\title{
Distilling angular momentum nonclassical states in trapped ions
}

\author{
B. Militello and A. Messina \\ INFM, MIUR, and Dipartimento di Scienze Fisiche ed Astronomiche dell'Università di Palermo, Via Archirafi, 36, \\ I-90123 Palermo, Italy
}

(Received 14 May 2004; published 23 September 2004)

\begin{abstract}
In the spirit of quantum nondemolition measurements, we show that by exploiting suitable vibronic couplings and repeatedly measuring the atomic population of a confined ion, it is possible to distill center-of-mass vibrational states with a well-defined square of angular momentum or, alternatively, angular momentum projection Schrödinger cat states.
\end{abstract}

DOI: 10.1103/PhysRevA.70.033408

PACS number(s): 42.50.Vk, 32.80.Pj, 03.65.Xp, 03.65.Ta

\section{INTRODUCTION}

The harmonic oscillator is a very archetypical and basic system both in quantum and classical physics. In fact, not only does it provide a good description of a physical system "moving" nearby a minimum of its potential energy, but in addition, it describes the independent modes of the electromagnetic field, throwing a bridge between (quantum) mechanics and (quantum) electrodynamics.

Today it is possible to confine an ion into a Paul trap in such a way that its center of mass behaves as a threedimensional harmonic oscillator [1,2]. A trapped ion, besides these vibrational degrees of freedom, possesses fermionic dynamical variables too, related to its internal state.

In such a physical context a wide variety of schemes for generating both classical and nonclassical states have been proposed and practically realized [1-4]. We mention here coherent, Fock, and squeezed states [5], and quantum superposition of states involving entanglement between the internal degrees of freedom (the atom) and the center-of-mass quasiclassical motion (two motion coherent states playing the role of the two cats' conditions "alive" and "dead" are considered) [6]. Moreover, coherent superpositions of macroscopically distinguishable states, often referred to as "Schrödinger cat" states too, have been reported. In particular it has been proposed that the generation of the superposition of two harmonic-oscillator energy eigenstates is characterized by opposite angular momentum projections corresponding to clockwise and counterclockwise center-ofmass motions $[7,8]$. Such a generation scheme, assuming that the trapped particle is completely "isolated," involves a long time interaction. Moreover, it requires a high degree of accuracy in chronological control of the experiment, in the sense that the system is described by the "cat" state during a very small time interval. Such critical points may be overcome by exploiting distillation processes [9] largely used in applications for quantum technology [10], i.e., quantum computation, quantum information, and quantum teleportation.

Generally speaking, distilling a state means nothing but extracting it from the initial state of the system, providing in some sense the realization of projection operators. A wide class of distillation processes are based on the idea that a physical system, the slave $(S)$, in interaction with a repeatedly measured one, the master $(M)$, undergoes a nonunitary evolution provoking the decay of most of the quantum states (the "residual") in favor of a few preserved ones (the "distillate") [11]. The relevant selection rule is related to both the specific master-slave interaction and the master measurement results. As a good example we mention quantum nondemolition measurements (QND) [12,13], largely used, for instance, in trapped ions for generating Fock states [14-16].

In this paper we give the general sketch of a distillation strategy developing the analysis in the specific framework of a wide class of vibronic couplings, which are realizable in the context of trapped ions. The statement of this general approach is then exploited in different applications. After revisiting the QND scheme, the possibility of generating a superposition of those Fock states whose quantum numbers correspond to "perfect squares" is proved. The crucial result of this paper is presented in the third section, wherein the previously mentioned generation of angular momentum Schrödinger cat states in two-dimensional traps is reported. The class of distilled cats turns out to be more general than that generated by the method in Ref. [8]. Indeed, in our case, a higher level of controllability of the quantum phase between the two terms of the superposition is obtained. The efficiency and the fidelity of the method are discussed and shown to be good enough. In the fourth section the distillation procedure is applied to a three-dimensional isotropically trapped ion, providing the possibility of generating eigenstates of the square of the orbital (i.e., related to the centerof-mass motion) angular momentum. Finally, in the last section, some conclusive remarks are given.

\section{DISTILLATION PROCESSES IN TRAPPED IONS}

In a Paul trap, a time-dependent inhomogeneous (quadrupolar) field is able to force a charged particle to move approximately as a harmonic oscillator. Hence, an ion confined in such a device provides a compound system possessing both fermionic (electronic) and bosonic (vibrational) degrees of freedom. The first ones describe the internal state of the ion, i.e., the motion of the electrons around the nucleus, and in most of the cases may be represented as a two-level system. The other degrees of freedom describe the oscillatory motion of the ion center of mass. The relevant unperturbed Hamiltonian is expressible as $(\hbar=1)$ 


$$
\hat{H}_{0}=\sum_{i=x, y, z} \nu_{i} \hat{a}_{\dagger}^{i} \hat{a}_{i}+\frac{\omega_{0}}{2} \hat{\sigma}_{3},
$$

where $\nu_{i}$ are the center-of-mass harmonic-oscillator frequencies, $\hat{a}_{i}\left(\hat{a}_{i}^{\dagger}\right)$ are the related annihilation (creation) operators, $\omega_{0}$ is the Bohr frequency between the two atomic levels considered, and $\hat{\sigma}_{3}$ is the diagonal Pauli operator.

Acting upon the system through laser fields, it is possible to implement a wide variety of vibronic couplings whose features depend on the laser frequencies, wavelengths, polarizations, and strengths. Generally speaking, the $\hat{H}_{0}$-interaction picture Hamiltonian model evaluated in the rotating wave approximation (RWA) turns out to be time independent and expressible as $[1,3,4]$

$$
\hat{H}_{v}=\gamma \hat{\Omega} \hat{\sigma}_{+}+\text {H.c. },
$$

where $\hat{\sigma}_{+}=|+\rangle\langle-|\left(\hat{\sigma}_{-}=|-\rangle\langle+|\right)$is the Pauli raising (lowering) operator, with $| \pm\rangle$ being the internal ionic states, and $\gamma$ is a positive coupling constant related to laser intensities and initial phases. The generic time-independent vibrational operator $\hat{\Omega}$ is a function of the annihilation and creation operators $\left\{\hat{a}_{i}\right\} \cup\left\{\hat{a}_{i}^{\dagger}\right\}$. Its specific form is determined once the specific laser-field configuration is given [3].

Assume the fermionic part of the compound system $(M)$ is initially in the state $|+\rangle$, hence starting with the density operator

$$
\hat{\rho}=\hat{\rho}_{v}|+\rangle\langle+|,
$$

$\hat{\rho}_{v}$ being the initial vibrational state (the initial state of $S$ ). Let the system evolve under the action of the Hamiltonian $\hat{H}_{v}(M-S$ interaction) for a time $\tau$, and then measure the internal ionic state. Assume the system is found in $|+\rangle$, and then, again let the system evolve in accordance with $\hat{H}_{v}$ for a time $\tau$, and measure the fermionic state finding it in $|+\rangle$, and so on $N$ times.

Under these assumptions, the system undergoes the nonunitary evolution described by

$$
\hat{W}_{+}^{(N)}(\tau) \equiv \aleph_{N}\left[|+\rangle\langle+| e^{-i \hat{H}_{v} \tau}\right]^{N}|+\rangle\langle+|,
$$

which may be cast in the form

$$
\hat{W}_{+}^{(N)}(\tau)=\aleph_{N}[\hat{V}(\tau)]^{N}|+\rangle\langle+|
$$

with

$$
\hat{V}(\tau) \equiv\left\langle+\left|e^{-i \hat{H}_{v} \tau}\right|+\right\rangle
$$

and $\aleph_{N}=\left[\Pi_{k=1}^{N} \sqrt{\wp_{k}}\right]^{-1}$, with $\wp_{k}$ being the probability of finding the ion into the state $|+\rangle$ at the $k$ th measurement step.

It is straightforward to prove that

$$
\begin{gathered}
\hat{H}_{v}^{2}=\gamma^{2}\left(\hat{\Omega}^{\dagger} \hat{\Omega}|-\rangle\left\langle-\left|+\hat{\Omega} \hat{\Omega}^{\dagger}\right|+\right\rangle\langle+|\right), \\
\hat{H}_{v}^{2 n}=\gamma^{2 n}\left[\left(\hat{\Omega} \hat{\Omega}^{\dagger}\right)^{n}|-\rangle\left\langle-\left|+\left(\hat{\Omega} \hat{\Omega}^{\dagger}\right)^{n}\right|+\right\rangle\langle+|\right],
\end{gathered}
$$

from which immediately follows

$$
\begin{gathered}
\left\langle+\left|\hat{H}_{v}^{2 n+1}\right|+\right\rangle=\hat{0}, \\
\left\langle+\left|\hat{H}_{v}^{2 n}\right|+\right\rangle=\gamma^{2 n}\left(\hat{\Omega} \hat{\Omega}^{\dagger}\right)^{n} .
\end{gathered}
$$

On the basis of these results, the unitary evolution operator associated with $\hat{H}_{v}$,

$$
e^{-i \hat{H}_{v} \tau}=\sum_{n=0}^{\infty}(-1)^{n} \frac{\hat{H}_{v}^{2 n} \tau^{2 n}}{(2 n) !}-i \sum_{n=0}^{\infty}(-1)^{n} \frac{\hat{H}_{v}^{2 n+1} \tau^{2 n+1}}{(2 n+1) !},
$$

restricted to the $|+\rangle$ master state, furnishes

$$
\hat{V}(\tau)=\cos \left(\gamma \tau \sqrt{\hat{\Omega} \hat{\Omega}^{\dagger}}\right) .
$$

Such a nonunitary operator is a real function of the Hermitian non-negative operator $\hat{\Omega} \hat{\Omega}^{\dagger}$, and hence is Hermitian too, and its eigensolution problem is strictly related to that of $\hat{\Omega} \hat{\Omega}$ (same eigenstates, different eigenvalues).

Let us denote by $\left|\omega_{k}\right\rangle$ and $\omega_{k}$ the eigenstates and the eigenvalues of $\hat{\Omega} \hat{\Omega}^{\dagger}$, respectively, and accordingly give the spectral decomposition $\hat{\Omega} \hat{\Omega}^{\dagger}=\Sigma_{k} \omega_{k}\left|\omega_{k}\right\rangle\left\langle\omega_{k}\right|$. Choose the interaction time $\tau$ in such a way that for some $k$ it results $\left|\cos \left(\gamma \tau \sqrt{\omega_{k}}\right)\right|=1$.

Thus, for large enough $N$ it turns out

$$
\begin{gathered}
\cos ^{N}\left(\gamma \tau \sqrt{\omega_{k}}\right)=(-1)^{l_{k} \cdot N}, \quad k \in I(\tau), \\
\cos ^{N}\left(\gamma \tau \sqrt{\omega_{k}}\right) \approx 0, \quad k \notin I(\tau),
\end{gathered}
$$

where

$$
I(\tau) \equiv\left\{k: \gamma \tau \sqrt{\omega_{k}}=l_{k} \pi, \quad l_{k} \in \mathbb{Z}\right\} .
$$

Finally, one has the following nonunitary action on the compound system:

$$
\hat{W}_{+}^{(N)}(\tau) \approx \aleph_{N}|+\rangle\left\langle+\left|\sum_{k \in I(\tau)}(-1)^{l_{k} \cdot N}\right| \omega_{k}\right\rangle\left\langle\omega_{k}\right|,
$$

which is equivalent to the following nonunitary action on the slave:

$$
\hat{V}^{N}(\tau) \approx \sum_{k \in I(\tau)}(-1)^{l_{k} \cdot N}\left|\omega_{k}\right\rangle\left\langle\omega_{k}\right| \equiv e^{-i \hat{P} \hat{P}_{d} \hat{G} \hat{P}_{d}} \hat{P}_{d},
$$

with $\hat{G}$ being a suitable Hermitian operator whose restriction, $\hat{P}_{d} \hat{G} \hat{P}_{d}$ to the subspace $\hat{P}_{d}=\Sigma_{k \in I(\tau)}\left|\omega_{k}\right\rangle\left\langle\omega_{k}\right|$, generates a unitary transformation into the target subspace. When all $l_{k} \cdot N$ are even, it turns out that $e^{-i \hat{P}_{d} \hat{G} \hat{P}_{d}}=\hat{1}$, and hence $\hat{V}^{N}(\tau) \approx \hat{P}_{d}$.

It is worth noting that in order to obtain the final result of the distillation process we do not need detailed knowledge of the dynamics induced by the Hamiltonian in Eq. (2), but just the diagonalization of the positive Hermitian operator $\hat{\Omega} \hat{\Omega}^{\dagger}$. This simplification shall reveal this to be very effective and useful.

It deserves to be remarked that the distillation is a conditional procedure in the sense that its success depends on $N$ stochastic events. In other words, it is required that the electronic system $(M)$, at each measurement act, is always found into the upper level $|+\rangle$. Otherwise the procedure fails. 
Moreover, the procedure is substantially the realization of a projection operator, hence, for the process being successful, the distilled states should be present in the initial vibrational condition $\hat{\rho}_{v}$. Both these problematic aspects of the method are solved considering that, as in the case of QND, the joint probability of finding the master system in its initial state at each step $\left(\Pi_{k=1}^{N} \wp_{k}\right)$ tends, in the limit of $N \rightarrow \infty$, to the probability of finding the target state (i.e., the "distillate") into the initial slave state (see Appendix A); that is,

$$
\prod_{k=1}^{N} \wp_{k} \rightarrow \operatorname{Tr}_{S}\left\{\hat{\rho}_{v} \hat{P}_{d}\right\}
$$

with $\operatorname{Tr}_{S}$ being the trace operation over the slave degrees of freedom.

The quantity in Eq. (16) expresses the efficiency of the distillation process, that is the probability of distillation success. Incidentally, Eq. (16) explains also the fact that QND may be used in trapped ions both as a strategy for generating states and for measuring vibrational state populations [14].

\section{A. Quantum nondemolition measurements of single Fock states}

Let us consider, as a specific example of this theory, the standard quantum nondemolition measurements.

Consider a laser field directed, for instance, along $x$, and tuned to the atomic Bohr frequency $\omega_{0}$. The relevant vibronic coupling has the form [3]

$$
\hat{H}_{v}=\gamma f\left(\hat{a}_{x}^{\dagger} \hat{a}_{x}, \eta_{x}\right) \hat{\sigma}_{+}+\text {H.c. },
$$

where

$$
f\left(\hat{a}_{x}^{\dagger} \hat{a}_{x}, \eta_{x}\right) \equiv e^{-\eta_{x}^{2} / 2} \sum_{l=0}^{\infty} \frac{\left(i \eta_{x}\right)^{2 l}}{(l !)^{2}} \hat{a}_{x}^{\dagger l} \hat{a}_{x}^{l}=\sum_{n=0}^{\infty} L_{n}^{(0)}\left(\eta_{x}^{2}\right)|n\rangle\langle n|,
$$

where $L_{n}^{(0)}\left(\eta_{x}^{2}\right)$ is a Laguerre polynomial, with $\eta_{x}$ being the Lamb-Dicke parameter defined as the ratio between the dimension of the oscillations of the ion center of mass in its ground vibrational state, and the laser field wavelength. Referring to Eq. (2) we have $\hat{\Omega}=\hat{\Omega}^{\dagger}=f\left(\hat{a}_{x}^{\dagger} \hat{a}_{x}, \eta_{x}\right)$, so that it results

$$
\hat{V}(\tau)=\cos \left[\gamma \tau f\left(\hat{a}_{x}^{\dagger} \hat{a}_{x}, \eta_{x}\right)\right] .
$$

It is easy to see that when $\eta_{x} \ll 1$ the function $f\left(n, \eta_{x}\right)$ approaches unity for any $n$, otherwise such a function is strongly nonlinear, and it turns out that different values of $n$ give rise to incommensurable values of $f$. Therefore, it is possible to choose the interaction time $\tau$ in such a way that $\gamma \tau f\left(\bar{n}_{x}, \eta_{x}\right)=2 \pi$, and $\gamma \tau f\left(\bar{n}_{x}, \eta_{x}\right) \neq l_{n} \pi$ for any $n \neq \bar{n}$, obtaining, for large enough $N$,

$$
\begin{gathered}
\cos ^{N}\left[\gamma \tau f\left(\eta_{x}, \bar{n}\right)\right]=1, \\
\cos ^{N}\left[\gamma \tau f\left(\eta_{x}, n\right)\right] \approx 0, \quad n \neq \bar{n} .
\end{gathered}
$$

This provides the decay of the Fock states $|n \neq \bar{n}\rangle$ in favor of the selected state $|\bar{n}\rangle$.

\section{B. Distillation of "perfect-square" Fock states}

As another application, consider the action of a laser field again directed along $x$ but tuned to the first blue sideband, i.e., tuned to the frequency $\omega_{0}+\nu_{x}$. In the Lamb-Dicke limit, i.e., assuming $\eta_{x} \ll 1$, the interaction picture Hamiltonian model turns out to be approximately the anti-JaynesCummings model

$$
\hat{H}_{v}=\gamma \hat{a}_{x}^{\dagger} \hat{\sigma}_{+}+\text {H.c. }
$$

This means that $\hat{\Omega}=\hat{a}_{x}^{\dagger}$. Therefore, the relevant $\hat{V}(\tau)$ is

$$
\hat{V}(\tau)=\cos \left(\gamma \tau \sqrt{\hat{a}^{\dagger} \hat{a}}\right) .
$$

It is straightforward to prove that, if $\gamma \tau=2 \pi$ is chosen, it results in

$$
\begin{gathered}
\cos ^{N}(\gamma \tau \sqrt{n})=1 \quad \text { if } n \text { is a square, } \\
\cos ^{N}(\gamma \tau \sqrt{n}) \approx 0 \quad \text { otherwise. }
\end{gathered}
$$

Therefore, the distillation procedure extracts the perfect square Fock states provoking the decay of all nonperfect square number states.

\section{DISTILLING TWO-DIMENSIONAL ANGULAR MOMENTUM SCHRÖDINGER CATS}

Let us now go through one of the two main results of this paper. It concerns the distillation of superpositions of vibrational states corresponding to bidimensional trapped ion center-of-mass motions characterized by the well-defined vibrational total excitation number and opposite projections of the angular momentum. In this sense we succeed in distilling angular momentum Schrödinger cat states. A scheme for generating such kinds of superpositions has been already proposed [8]. In fact, starting from a specific initial condition (a Fock state), and subjecting the system to the action of a bilinear two-mode Jaynes-Cummings-like Hamiltonian model $\left(\propto \hat{a}_{x} \hat{a}_{y} \hat{\sigma}_{+}+\right.$H.c. $)$, at some specific instants of time the ion center of mass is found in the mentioned cat. Nevertheless, such a procedure requires a very high degree of accuracy in temporal control of the experiment. We propose here to exploit the general distillation strategy to extract the same state generated with the recalled scheme. Moreover, the class of states we succeed in generating is wider than the one previously mentioned. In fact, the phase relation between the two terms of the superposition is, in our case, very easy to control.

Consider an isotropic bidimensional Paul trap, that is, assume, for instance, $\nu_{x}=\nu_{y} \equiv \nu$ and neglect the motion along $z$. Act on the system simultaneously through two laser beams both tuned to the second red sideband $\omega_{0}-2 \nu$. Let the two lasers be responsible for the same coupling strength. The relevant interaction Hamiltonian is [8]

$$
\hat{H}_{v}=\gamma\left(\hat{a}_{x}^{2}+\hat{a}_{y}^{2}\right) \hat{\sigma}_{+}+\text {H.c. }
$$

Observe now that the operator $\hat{\Omega} \hat{\Omega}^{\dagger}$ may be cast in the form 


$$
\hat{\Omega} \hat{\Omega}^{\dagger}=\sum_{j, k=x, y} \hat{a}_{j}^{2} \hat{a}_{k}^{\dagger 2}=\hat{N}_{T}^{2}+4 \hat{N}_{T}-\hat{L}_{z}^{2}+4,
$$

with $\hat{N}_{T}=\hat{a}_{x}^{\dagger} \hat{a}_{x}+\hat{a}_{y}^{\dagger} \hat{a}_{y}$, and $\hat{L}_{z}=i\left[\hat{a}_{x} \hat{a}_{y}^{\dagger}-\hat{a}_{y} \hat{a}_{x}^{\dagger}\right]$

On the basis of Eq. (25) it turns out that, suitably choosing $\gamma \tau$, the distillation process in this case may preserve those states characterized by specific excitation number $\left(n_{T}\right)$ and angular momentum projection $(m)$, denoted by $\left|n_{T}, m\right\rangle$, such that $\gamma \tau \sqrt{n_{T}^{2}+4 n_{T}-m^{2}+4}=l_{m} \pi, l_{m}$ being an integer. Observe now that both $m$ and $-m$ eventually satisfy such a relation. Moreover, that in general for different $n_{T}$ and/or the absolute value of $m$ the relevant square roots result to be incommensurable. Therefore, experimental details, like coupling strength $(\gamma)$, interaction time $(\tau)$, etc., may be adjusted to project the system into the bidimensional subspace generated by $\left\{\left|n_{T}, \pm m\right\rangle\right\}$, in accordance with Eq. (12). Thus, if the initial condition is a state whose overlaps with $\left|n_{T}, \pm m\right\rangle$ have the same modulus, the distilling procedure returns the $50 \%$ superposition of such angular momentum eigenstates.

As a very specific example consider the trapped ion prepared in the Fock state possessing $n_{T}=4$ vibrational excitations and directed along $x,\left|n_{x}=4, n_{y}=0\right\rangle$. Such a state is expressible as a superposition of the states $\left|n_{T}=4, m\right\rangle$ with $m$ $=0, \pm 2, \pm 4$. It is straightforward to calculate $\left\langle n_{T}=4, m\right.$ $= \pm 4\left|n_{x}=4, n_{y}=0\right\rangle=\frac{1}{4}$, and to verify that the "square roots" corresponding to different absolute values of $m$ are incommensurable $(2 \sqrt{5}, 4 \sqrt{2}, 6$, for $m= \pm 4, \pm 2,0$, respectively). Adjusting $\tau$ in such a way that $2 \sqrt{5} \gamma \tau=2 \pi$, one easily distillates, up to a global phase factor, the superposition

$$
\left|\psi_{\text {dist }}\right\rangle=\frac{1}{\sqrt{2}}\left[\left|n_{T}=4, m=4\right\rangle+\left|n_{T}=4, m=-4\right\rangle\right],
$$

which, as announced, is just an angular momentum Schrödinger cat superposition.

Straightforwardly one obtains that the efficiency of the distillation is $1 / 4^{2}+1 / 4^{2}=\frac{1}{8}$, and that, after $N=5$ steps, the Schrödinger cat state is distilled with a $5 \%$ error.

In this specific example the relative phase factor between the two states of the superposition turns out to be 1 . Nevertheless, by selecting a different direction of the initial motion, it is possible to change the relative phase between the two states in the initial condition and hence in the distilled superposition. In particular, denoting by $\theta$ the angle between the direction of the initial motion and the $x$ axis, it results that the relative phase between the two states of the distilled superposition is $2 n_{T} \theta$, giving

$$
\left|\psi_{\text {dist }}\right\rangle=\frac{1}{\sqrt{2}}\left[\left|n_{T}=4, m=4\right\rangle+e^{i 8 \theta}\left|n_{T}=4, m=-4\right\rangle\right] .
$$

To prove such an assertion it is enough to consider that both a Fock state directed along the direction rotated of $\theta$ around $z$ with respect to the $x$ axis, denoted by $\left|n_{\theta}\right\rangle$, and the angular momentum eigenstates of maximum projection $\left|n_{T}, m= \pm n_{T}\right\rangle$, are $\mathrm{SU}(2)$ states [17] expressible as

$$
\left|\mu, j=\frac{n_{T}}{2}\right\rangle=\frac{1}{\left(1+|\mu|^{2}\right)^{n_{T} / 2}} \sum_{k=0}^{n_{T}}\left(\begin{array}{c}
n_{T} \\
k
\end{array}\right)^{1 / 2} \mu^{k}\left|n_{T}-k, k\right\rangle .
$$

For $\mu=\tan \theta$ one has "rotated Fock states" $\left(\left|n_{\theta}\right\rangle=\mid \mu\right.$ $\left.\left.=\tan \theta, j=n_{T} / 2\right\rangle\right)$, that is an eigenstate of the number operator associated with the annihilation operator $\hat{a}_{\theta}$ $\equiv 1 / \sqrt{2}\left[\cos \theta \hat{a}_{x}+\sin \theta \hat{a}_{y}\right]$. For $\mu= \pm i$ one has maximum projection angular momentum eigenstates $\left|n_{T}, m= \pm n_{T}\right\rangle=\mid \mu$ $\left.= \pm i, j=n_{T} / 2\right\rangle$ [7], which may be directly verified. Their overlap is easily appraisable (see Appendix B) and it turns out that

$$
\left\langle n_{T}, m= \pm n_{T}\right| n_{\theta}=\frac{1}{2^{n_{T} / 2}} e^{\mp i n_{T} \theta} .
$$

Therefore, as anticipated, the relative phase between the two angular momentum eigenstates in both the initial and the distilled states is $\xi(\theta)=2 n_{T} \theta$. The efficiency $\left(\frac{1}{8}\right)$ and the agreement (5\% after $N=5$ steps) are the same as in the particular case $\theta=0$.

\section{DISTILLING THREE-DIMENSIONAL ANGULAR MOMENTUM EIGENSTATES}

The last application we present in this paper concerns the possibility of generating tridimensional states of motion characterized by a well-defined vibrational total excitation number and square of angular momentum. To this end, consider an isotropic tridimensional Paul trap $\left(\nu_{x}=\nu_{y}=\nu_{z} \equiv \nu\right)$, which may be easily realized adding to the standard quadrupolar sinusoidally time-dependent electric field, a quadrupolar static electric field [18].

The action of three orthogonally propagating lasers tuned to the second red sideband produces the tridimensional generalization of the coupling term in Eq. (24), i.e., the vibronic coupling in Eq. (2) with

$$
\hat{\Omega}=\hat{a}_{x}^{2}+\hat{a}_{y}^{2}+\hat{a}_{z}^{2} .
$$

Few algebraic manipulations give

$$
\hat{\Omega} \hat{\Omega}^{\dagger}=\sum_{j, k=x, y, z} \hat{a}_{j}^{2} \hat{a}_{k}^{\dagger 2}=\hat{N}_{T}^{2}+5 \hat{N}_{T}-\vec{L}^{2}+6,
$$

being that $\hat{N}_{T}=\sum_{j=x, y, z} \hat{a}_{j}^{\dagger} \hat{a}_{j}$, and $\vec{L}=\left(\hat{L}_{x}, \hat{L}_{y}, \hat{L}_{z}\right)$ with $\hat{L}_{l}$ $=i\left[\hat{a}_{j} \hat{a}_{k}^{\dagger}-\hat{a}_{k} \hat{a}_{j}^{\dagger}\right]$ for $(j, k, l)=(x, y, z)$ and cyclics.

As in the bidimensional case, the incommensurability of the square roots $\sqrt{n_{T}^{2}+5 n_{T}-l(l+1)+6}$ for different values of $n_{T}$ and $l$ ensures the possibility of extracting prefixed states. Here $n_{T}$ and $l$ are the vibrational total excitation and the angular momentum square quantum numbers. As a consequence, a single value of square of angular momentum may be selected. On the other hand, the circumstance that the eigenvalues of $\hat{L}_{z}$ (or of any other component) do not appear in the square root implies no selection of its eigenstates. Therefore, in general, the result of the distillation process is a generic linear combination, 


$$
\left|\psi_{\text {dist }}\right\rangle=\sum_{m=-l}^{l} c_{m}\left|n_{T}, l, m\right\rangle
$$

involving states possessing well-defined (i.e., the same for all) $\hat{N}_{T}$ and $\vec{L}^{2}$ but different angular momentum projections.

As an example we mention that starting with the Fock state $\left|n_{x}=2, n_{y}=n_{z}=0\right\rangle$, and choosing $\tau$ such that $\gamma \tau 2 \sqrt{5}$ $=2 \pi$, it is possible to distillate the state $\left|n_{T}=2, l=0, m=0\right\rangle$ with an efficiency $\frac{1}{3}$.

In passing we observe that, since the target angular momentum is $l=0$, the distilled subspace turns out to be one dimensional without ambiguity for the projection $(m=0)$.

It is worth noting that angular momentum eigenstates are, in general, strongly entangled states in the Fock basis. Hence, their generation provides an effective strategy for tridimensional entangled-states preparation. Accordingly with our example, consider indeed the Fock-basis expansion of the distilled state,

$$
\left|n_{T}=2, l=0, m=0\right\rangle=\frac{1}{\sqrt{3}}[|2,0,0\rangle+|0,2,0\rangle+|0,0,2\rangle] .
$$

It turns out that the spherically symmetric angular momentum eigenstate considered, $\left|n_{T}=2, l=m=0\right\rangle$, is a strongly entangled state involving the three orthogonal motions of the ion center of mass, possessing the same structure as a $W$ state [19]. In this case, after $N=5$ steps one reaches the target state in Eq. (32) with less than $4 \%$ error.

The action of the two- and three-dimensional reported distillation processes may be in principle combined in order to realize tridimensional center-of-mass motion "Schrödinger cats" characterized by well-defined vibrational excitations and a square of angular momentum, but involving states with opposite projections of angular momentum.

\section{DISCUSSION AND CONCLUSIVE REMARKS}

In this paper we have presented a strategy for distilling single harmonic-oscillator nonclassical states having the form of superpositions of macroscopically distinguishable angular momentum eigenstates (namely, angular momentum Schrödinger cat states). We, moreover, succeeded, in the framework of the same distillation method, to synthesize states possessing, a well-defined square of angular momentum. The physical scenario wherein these results have been presented is that of a single trapped ion-whose center of mass moving in the trapping potential is the harmonic oscillator under scrutiny.

The general distillation strategy that we use, recalled in the second section, exploits repeated measurements on a system in interaction with the one where we want to extract some interesting states. In fact, in such a situation, the latter system undergoes a nonunitary evolution provoking the decay of the undesired states, keeping in life only a few states (the "distillate"). Giving an explicit expression of the nonunitary operator is not always a trivial job. Moreover, due to such a nonunitariness, in some cases it is impossible to per- form its spectral decomposition. Nevertheless, in the framework of the spin-boson interaction, and in particular, in the context of trapped ions, we succeed in providing a very manageable expression for the effective nonunitary operator acting upon the bosonic system (the center-of-mass motion) when the fermionic system (the atomic internal state) is repeatedly measured. Such a very expressive and useful result, given in Eq. (11), guarantees the possibility of obtaining the spectral decomposition of the nonunitary operator acting upon the system of interest, $\hat{V}(\tau)=\cos \left(\gamma \tau \sqrt{\hat{\Omega} \hat{\Omega}^{\dagger}}\right)$, tracing such a problem back to the spectral decomposition of the positive Hermitian operator $\hat{\Omega} \hat{\Omega}^{\dagger}$. Therefore, we have at our disposal a powerful tool to forecast and control (suitably setting the coupling strength, $\gamma$, and the interaction time, $\tau$ ) the result of the distillation process.

Exploiting the potentialities of Eq. (11), in the third section we have found that in a bidimensional isotropic trap a suitable two-mode vibronic coupling is responsible for distilling superpositions of vibrational states possessing opposite angular momentum projections. Moreover, as reported in the fourth section, the analogous (mutatis mutandis) interaction in an isotropic tridimensional Paul trap renders it possible to extract center-of-mass motion states possessing a well-defined excitation number and square of angular momentum. In a specific mentioned example, such a state turns out to have the structure of a $W$ state.

As a conclusive remark we state that, since in general the number of steps required to distillate the target subspace is very small (in the considered examples $N=5$ to have $95 \%$ fidelity), the duration of the experiment turns out to be short enough to legitimize neglecting decoherence.

\section{APPENDIX A: DISTILLATION EFFICIENCY}

In this Appendix we prove the limit expressed by Eq. (16). Assume the system prepared, for simplicity, into the pure state $\left|\psi_{0}\right\rangle=\left|\varphi_{0}\right\rangle|+\rangle,\left|\varphi_{0}\right\rangle$ being the initial vibrational state. The probability of finding the Master system (fermionic degrees of freedom) in $|+\rangle$ after the unitary $M-S$ interaction is $\wp_{1}=\left\|\left\langle+\left|e^{-i \hat{H}_{v} \tau}\right| \psi_{0}\right\rangle\right\|^{2}=\| \hat{V}(\tau)\left|\varphi_{0}\right\rangle\left\|^{2},\right\| \cdot \|^{2} \equiv|\langle\cdot \mid \cdot\rangle|^{2}$ denoting the relevant Hilbert space "norma." The resulting "collapsed" state is $\left|\psi_{1}\right\rangle=\left(1 / \sqrt{\wp_{1}}\right)\left[\hat{V}(\tau)\left|\varphi_{0}\right\rangle\right]|+\rangle$. Immediately after the second step one obtains $\wp_{2}=\left\|\left\langle+\left|e^{-i \hat{H}_{v} \tau}\right| \psi_{1}\right\rangle\right\|^{2}$ $=1 / \wp_{1} \|\left[\hat{V}^{2}(\tau)\left|\varphi_{0}\right\rangle\right] \|^{2}$, and $\left|\psi_{2}\right\rangle=\left(1 / \sqrt{\wp_{1} \wp_{2}}\right)\left[\hat{V}^{2}(\tau)\left|\varphi_{0}\right\rangle\right]|+\rangle$. In general it is

$$
\begin{gathered}
\wp_{n}=\frac{1}{\wp_{0} \wp_{1} \cdots \wp_{n-1}} \| \hat{V}^{n}(\tau)\left|\varphi_{0}\right\rangle \|^{2}, \\
\left|\psi_{n}\right\rangle=\frac{1}{\sqrt{\wp_{0} \wp_{1} \cdots \wp_{n}}} \hat{V}^{n}\left|\varphi_{0}\right\rangle|+\rangle .
\end{gathered}
$$

From Eq. (A1) it follows that 


$$
\prod_{k=1}^{N} \wp_{k}=\wp_{N} \prod_{k=1}^{N-1} \wp_{k}=\| \hat{V}^{N}(\tau)\left|\varphi_{0}\right\rangle \|^{2} .
$$

Considering the limit $N \rightarrow \infty$, we can take advantage of Eq. (15) easily obtaining

$$
\prod_{k=1}^{N} \wp_{k} \approx \| \hat{P}_{d}\left|\varphi_{0}\right\rangle \|^{2},
$$

which expresses the same content of Eq. (16).

\section{APPENDIX B: OVERLAP BETWEEN SU(2) STATES}

In this Appendix we give the general expression of the overlap between two two-mode SU(2) states, and calculate the phase between the maximum projection angular momentum eigenstates and the rotated Fock states.
From the definition in Eq. (28), the overlap is easily calculated as

$$
\left\langle\mu_{1}, j_{1} \mid \mu_{2}, j_{2}\right\rangle=\frac{\left(1+\mu_{1}^{*} \mu_{2}\right)^{2 j}}{\left(1+\left|\mu_{1}\right|^{2}\right)^{j_{1}}\left(1+\left|\mu_{2}\right|^{2}\right)^{j_{2}}} \delta_{j_{1}, j_{2}} .
$$

In the case $\mu_{1}= \pm i$ and $\mu_{2}=\tan \theta$ one obtains

$$
\begin{aligned}
\left\langle\mu_{1}\right. & \\
& = \pm i, j\left|\mu_{2}=\tan \theta, j\right\rangle=\frac{(1 \mp \tan \theta)^{2 j}}{2^{j}\left(1+\tan ^{2} \theta\right)^{j}} \\
& =\frac{\left(\sqrt{1+\tan ^{2} \theta} e^{\mp i \theta}\right)^{2 j}}{2^{j}\left(1+\tan ^{2} \theta\right)^{j}}=\frac{e^{\mp i 2 j \theta}}{2^{j}} .
\end{aligned}
$$

Therefore, the phase difference between the coefficients of the $\mathrm{SU}(2)$ states with $\mu=+i$ and $\mu=-i$ contained in $|\mu=\tan \theta, j\rangle$ results in $4 j \theta=4\left(n_{T} / 2\right) \theta=2 n_{T} \theta$.
[1] D. Leibfried et al., Rev. Mod. Phys. 75, 281 (2003).

[2] P. K. Ghosh, Ion Traps (Clarendon, Oxford, 1995).

[3] W. Vogel and S. Wallentowitz, in Coherence and Statistics of Photons and Atoms, edited by Jan Perina (Wiley, New York, 2001).

[4] D. J. Wineland et al., J. Res. Natl. Inst. Stand. Technol. 103, 259 (1998).

[5] D. M. Meekhof et al., Phys. Rev. Lett. 76, 1796 (1996).

[6] C. Monroe et al., Science 272, 1131 (1996).

[7] S. Maniscalco et al., J. Mod. Opt. 47, 2113 (2000); Phys. Rev. A 61, 053806 (2000).

[8] S. Maniscalco et al., J. Mod. Opt. 48, 2065 (2001).

[9] C. H. Bennett et al., Phys. Rev. Lett. 76, 722 (1996); 78, 2031 (1997); Phys. Rev. A 54, 3824 (1996); J. I. Cirac, A. K. Ekert, and C. Macchiavello, Phys. Rev. Lett. 82, 4344 (1999); see also Refs. [10], and references therein.

[10] For reviews, see A. Galindo and M. A. Martin-Delgado, Rev. Mod. Phys. 74, 347 (2002); The Physics of Quantum Information, edited by D. Bouwmeester, A. Ekert, and A. Zeilinger (Springer, Berlin, 2000); M. A. Nielsen and I. L. Chuang,
Quantum Computation and Quantum Information (Cambridge University Press, Cambridge, 2000).

[11] H. Nakazato, T. Takazawa, and K. Yuasa, Phys. Rev. Lett. 90, 060401 (2003)

[12] V. B. Braginsky and F. Ya. Khalili, Quantum Measurement (Cambridge University Press, Cambridge, 1992).

[13] D. F. Walls and G. J. Milburn, Quantum Optics (Springer, Berlin, 1994).

[14] R. L. de Matos Filho and W. Vogel, Phys. Rev. Lett. 76, 4520 (1996).

[15] L. Davidovich et al., Phys. Rev. A 54, 5118 (1996).

[16] K. Wang et al., Phys. Rev. A 63, 043419 (2001).

[17] A. M. Perelomov, Commun. Math. Phys. 26, 222 (1972).

[18] P. E. Toschek, New Trends in Atomic Physics, edited by G. Grynberg and R. Stora (Elsevier Science, Amsterdam (1984), p. 282.

[19] D. M. Greenberger, M. A. Horne, A. Shimony, and A. Zeilinger, Am. J. Phys. 58, 1131 (1990); L. Hardy, Contemp. Phys. 39, 6, 419 (1998). 